\title{
Phase I study on the safety, pharmacokinetic profile, and efficacy of the combination of TSU-68, an oral antiangiogenic agent, and S-1 in patients with advanced hepatocellular carcinoma
}

\author{
Masafumi Ikeda • Shuichiro Shiina • Kohei Nakachi • Shuichi Mitsunaga • Satoshi Shimizu • \\ Yasushi Kojima • Hideki Ueno • Chigusa Morizane • Shunsuke Kondo • \\ Yasunari Sakamoto • Yoshinari Asaoka • Ryosuke Tateishi • Kazuhiko Koike • \\ Hitoshi Arioka • Takuji Okusaka
}

Received: 20 February 2014 / Accepted: 30 April 2014 /Published online: 15 May 2014

(C) The Author(s) 2014. This article is published with open access at Springerlink.com

Summary Purpose We aimed to investigate the recommended dose for the combination of TSU-68, a multiple-receptor tyrosine kinase inhibitor targeting vascular endothelial growth factor receptor-2 and platelet-derived growth factor receptor- $\beta$, and $\mathrm{S}-1$, an oral fluoropyrimidine, in patients with advanced hepatocellular carcinoma (HCC) based on its associated dose-limiting toxicity (DLT) frequency. We also determined the safety, tolerability, pharmacokinetics (PK), and efficacy of the combination treatment. Patients and methods Patients without any prior systemic therapy received $400 \mathrm{mg} /$ day TSU-68 orally and $80 \mathrm{mg} /$ day (level 1) or $100 \mathrm{mg} /$ day (level 2) S-1 for 4 or 2 weeks followed by a 2 - or 1 -week rest

M. Ikeda $(\bowtie) \cdot$ K. Nakachi $\cdot$ S. Mitsunaga $\cdot$ S. Shimizu $\cdot$ Y. Kojima

Department of Hepatobiliary and Pancreatic Oncology,

National Cancer Center Hospital East, 6-5-1 Kashiwanoha,

Kashiwa 277-8577, Japan

e-mail: masikeda@east.ncc.go.jp

S. Shiina

Department of Gastroenterology, Graduate School of Medicine,

Juntendo University, Tokyo, Japan

Y. Kojima

Department of Gastroenterology, National Center for Global Health and Medicine, Tokyo, Japan

H. Ueno • C. Morizane · S. Kondo • Y. Sakamoto • T. Okusaka Department of Hepatobiliary and Pancreatic Oncology,

National Cancer Center Hospital, Tokyo, Japan

S. Shiina $\cdot$ Y. Asaoka $\cdot$ R. Tateishi $\cdot$ K. Koike

Department of Gastroenterology, Graduate School of Medicine,

The University of Tokyo, Tokyo, Japan

H. Arioka

Department of Medical Oncology, Yokohama Rosai Hospital,

Yokohama, Japan period (groups $\mathrm{A}$ and $\mathrm{B}$, respectively). According to the treatment, patients progressed from level $1 \mathrm{~B}$ to level $2 \mathrm{~A}$, then level 2B. Safety and response rates were assessed. Results Eighteen patients were enrolled. Two patients at levels $1 \mathrm{~B}$ and $2 \mathrm{~A}$ but none at level $2 \mathrm{~B}$ showed DLTs. The common adverse drug reactions were a decrease in hemoglobin levels, hypoalbuminemia, and anorexia, which were mild in severity (grades 1-2). PK data from levels $1 \mathrm{~B}$ and $2 \mathrm{~A}$ indicated that the area under the curve for TSU-68 and 5-fluorouracil was unlikely to be affected by the combination treatment. Response rate, disease control rate, median time to progression, and median overall survival were $27.8 \%, 61.1 \%, 5.3$ months, and 12.8 months, respectively. Conclusion The recommended dose for advanced HCC should be $400 \mathrm{mg}$ /day TSU-68 and $100 \mathrm{mg} /$ day S-1 for 4 weeks followed by 2 -week rest.

Keywords Hepatocellular carcinoma · TSU-68 · S-1 · Chemotherapy · VCAM-1

\section{Introduction}

Hepatocellular carcinoma (HCC) is one of the most common cancers worldwide [1]. Surgical resection, percutaneous ethanol injection (PEI), or radiofrequency ablation (RFA) is often performed in patients with curable HCC, whereas transcatheter arterial chemoembolization (TACE) is the treatment of choice for patients with incurable HCC. Sorafenib is the standard chemotherapy for HCC $[2,3]$, but its efficacy is limited. Therefore, the search for novel medical therapies is essential for HCC management.

As HCC is a highly vascular tumor, a number of antiangiogenic agents have been tested for its treatment. TSU-68 
is a small-molecule, orally administered, multiple-receptor tyrosine kinase inhibitor of the vascular endothelial growth factor receptor-2 (VEGFR-2) and platelet-derived growth factor receptor- $\beta$ (PDGFR- $\beta$ ) [4-8]. TSU-68 demonstrated favorable efficacy and a good safety profile for HCC $[9,10]$. A phase III clinical trial of TSU-68 with TACE (ClinicalTrials.gov identifier NCT01465464 ORIENTAL study) is currently underway.

S-1 is an orally administered fluoropyrimidine anticancer drug that combines tegafur (FT), 5-chloro-2,4dihydroxypyridine (CDHP), and oteracil potassium (Oxo) in a molar concentration ratio of 1:0.4:1 [11]. CDHP is a competitive inhibitor of dihydropyrimidine dehydrogenase (DPD), a 5-fluorouracil (5-FU)-metabolizing enzyme expressed in the liver. Therefore, prolonged effective concentrations of 5-FU in the plasma and tumor tissue can be achieved via successful inhibition of DPD by CDHP. Oxo, a competitive inhibitor of orotate phosphoribosyltransferase, inhibits the phosphorylation of 5-FU in the gastrointestinal tract, thereby reducing serious 5-FU-related gastrointestinal toxicity. Clinically, S-1 has been shown to be effective against various solid tumors. In Japan, the clinical development of chemotherapies for unresectable, advanced, or recurrent gastric cancers has progressed for many years, and many clinical studies have been conducted. S-1 has showed favorable efficacy and a good safety profile for HCC in a phase I/II study [12]. A phase III clinical trial of S-1 for HCC (Japicdoc identifier JapicCTI-090920 S-CUBE trial) is underway.

Many molecular targeted drugs and anticancer agents have been administered as combination therapies to improve efficacy in various types of cancer. In HCC, combination therapies of molecular targeted agents [13], a molecular-targeted agent and cytotoxic chemotherapy [14-18], and a molecular targeted agent and TACE have been developed [19, 20]. However, no combination therapy has been proven to improve survival [21]. TSU-68 and S-1 have been shown to be effective against HCC in a phase I/II study as single agents. Therefore, we investigated the TSU-68 plus S-1 combination.

This study investigated the recommended dose of TSU-68 plus S-1 in HCC based on the frequency of associated doselimiting toxicity (DLT). We determined safety, tolerability, pharmacokinetics (PK), and efficacy of the TSU-68 plus S-1 combination in patients with advanced HCC. We also evaluated the expression of endothelial cell markers in patients receiving TSU-68 plus S-1 combination therapy.

\section{Methods}

\section{Eligibility criteria}

The following patients were eligible: (1) patients with confirmed HCC according to histological studies or diagnostic imaging; (2) patients classified as having advanced disease if they were not eligible for or showed disease progression after surgery, PEI, RFA, or TACE; (3) patients with a minimum age of 20 years; (4) patients with an Eastern Cooperative Oncology Group performance status scale score of 0 or 1 ; (5) patients with measurable disease based on the Response Evaluation Criteria in Solid Tumors (RECIST) v1.0; and (6) patients with a white blood cell count of $\geq 3,000$ cells $/ \mathrm{mm}^{3}$ or neutrophil count of $\geq 1,500$ cells $/ \mathrm{mm}^{3}$, hemoglobin level of $\geq 9.0 \mathrm{~g} / \mathrm{dL}$, platelet count of $\geq 7.5 \times 10^{4}$ cells $/ \mathrm{mm}^{3}$, total bilirubin level of $2.0 \mathrm{mg} / \mathrm{dL}$, aspartate aminotransferase (AST) and alanine aminotransferase (ALT) levels $\leq$ upper limit of normal $\times 5$, albumin level of $\geq 3.0 \mathrm{mg} / \mathrm{dL}$, prothrombin timeinternational normalized ratio of $\leq 2.0$, and creatinine level $\leq$ upper limit of normal.

Patients were not eligible if they had received surgery, PEI, RFA, TACE, chemotherapy, or radiotherapy within 30 days before the start of this study. Patients were excluded if there was clinical evidence of central nervous system metastasis, severe cardiovascular disorder, hepatic encephalopathy, uncontrollable pleural effusion or ascites, or a serious infection. Patients requiring prophylactic variceal ligation or sclerotherapy were also excluded.

All patients provided written informed consent. The study was approved by the institutional review board of each of the 3 participating hospitals and was performed in accordance with the principles of the Declaration of Helsinki and Good Clinical Practice Guidelines.

\section{DLTs}

The following were considered as DLTs: (1) a hematological adverse drug reaction of grade 4 (grade 3 febrile neutropenia), (2) a non-hematological adverse drug reaction of grade 3 (excluding AST, ALT, alkaline phosphatase, gammaglutamyl transpeptidase, Na, and K levels), (3) AST and ALT levels of 10 times higher than the upper limit of normal values, (4) an adverse drug reaction requiring discontinuation of TSU-68 treatment for 15 consecutive days during a cycle, (5) an adverse drug reaction requiring discontinuation of S-1 treatment for 8 consecutive days during a cycle, and (6) an adverse drug reaction requiring discontinuation of TSU-68 plus S-1 treatment.

Study design and treatment

The present study was designed as an open-label phase I study. The patients received $400 \mathrm{mg}$ /day TSU-68 orally and one of the following doses of S-1: $50 \mathrm{mg} /$ day (level 0), $80 \mathrm{mg} /$ day (level 1), or $100 \mathrm{mg}$ /day (level 2). TSU-68 and S-1 were administered orally twice daily (after breakfast and supper). For S-1, dose levels were administered according to the following administration and resting schedules: for levels $0 \mathrm{~B}$, 
$1 \mathrm{~B}$, and $2 \mathrm{~B}, \mathrm{~S}-1$ was administered continuously for 28 days followed by a 14-day rest period, which completed a treatment cycle, and for levels $0 \mathrm{~A}, 1 \mathrm{~A}$, and $2 \mathrm{~A}$, a cycle of S-1 was defined as continuous administration of the drug for 14 days followed by a 7-day rest period. Drug administration was continued by repeating the cycle, unless one of the following occurred: progressive disease, unacceptable toxicity, withdrawal of patient consent, or termination of treatment at the attending physician's discretion. Treatment dose and duration started at level 1B, followed by progression to $2 \mathrm{~A}-2 \mathrm{~B}$ or $1 \mathrm{~A}-0 \mathrm{~A}$ in the event of DLTs (Fig. 1). The recommended dose was determined based on the treatment dose, duration, DLTs, toxicity, and efficacy.

\section{Assessment of efficacy and toxicity}

DLTs were recorded for each treatment level, and the rate of DLT development was determined. During each course of treatment, the tumor response was assessed according to RECIST v1.0 by using computed tomography or magnetic resonance imaging, with a maximum slice thickness of $5 \mathrm{~mm}$ [22]. Efficacy was evaluated in terms of the response rate (RR), disease control rate (DCR), median time to progression (TTP), and median overall survival (OS) time. TTP was defined as the time from the date of registration to the date when progressive disease was confirmed based on RECIST. OS was defined as the time from the date of registration to the date of death. Physical examination findings and results of serum and urine chemistry analyses were obtained at 1-week intervals for the first cycle and at 2-week intervals during the remaining cycles. Vital signs were assessed as necessary. The severity of all adverse events was evaluated according to the Common Terminology Criteria for Adverse Events, version 3.0.

Fig. 1 Administration schedule of the TSU-68 plus S-1 combination. Patients received $400 \mathrm{mg}$ /day TSU-68 and 50,80 or $100 \mathrm{mg} /$ day S-1. The initial dose and treatment duration for $\mathrm{S}-1$ were at level 1B followed by progression to levels $2 \mathrm{~A}-2 \mathrm{~B}$ in case of dose-limiting toxicity $(\mathrm{DLT}) \leq 2 / 6$ or levels $1 \mathrm{~A}-0 \mathrm{~A}$ in case of DLT $\geq 3 / 6$

\begin{tabular}{|c|c|}
\hline \multicolumn{2}{|c|}{ Level 2B } \\
4 weeks followed by a 2-week rest \\
\hline $\begin{array}{c}\text { BSA } \\
<1.25\end{array}$ & $80 \mathrm{mg} /$ day \\
\hline $\begin{array}{c}\text { BSA } \\
\geq 1.25-<1.5\end{array}$ & $100 \mathrm{mg} /$ day \\
\hline $\begin{array}{c}\text { BSA } \\
\geq 1.5\end{array}$ & $120 \mathrm{mg} /$ day \\
\hline $\begin{array}{c}\text { DLT } \\
2 / 6\end{array}$ \\
\begin{tabular}{|c|c|}
\hline \multicolumn{2}{|c|}{ Level $2 \mathrm{~A}$} \\
2 weeks followed by a 1 -week rest \\
\hline $\begin{array}{c}\text { BSA } \\
<1.25\end{array}$ & $80 \mathrm{mg} /$ day \\
\hline $\begin{array}{c}\text { BSA } \\
\geq 1.25-<1.5\end{array}$ & $100 \mathrm{mg} /$ day \\
\hline $\begin{array}{c}\text { BSA } \\
\geq 1.5\end{array}$ & $120 \mathrm{mg} /$ day \\
\hline
\end{tabular}
\end{tabular}

PK

We evaluated the PK of co-administered TSU-68 and S- 1 on the basis of their plasma concentrations and PK parameters obtained in 12 patients at levels $1 \mathrm{~B}$ and $2 \mathrm{~A}$. Blood samples were collected before dosing and at $1,2,3,4,6,9$, and $12 \mathrm{~h}$ on days 1 and 8 after dosing. TSU-68 plasma concentration was determined using a validated ultraviolet high-performance liquid chromatography method. Plasma concentrations of FT, 5-FU, CDHP, and Oxo were determined using a validated liquid chromatography-tandem mass spectrometry method. PK parameters, including maximum plasma concentration $\left(\mathrm{C}_{\max }\right)$, time to reach the maximum plasma concentration, area under curve (AUC), and plasma half-life, were calculated with the non-compartmental method using the PK analysis program WinNonlin Professional (Pharsight Corporation, St. Louis, MO).

\section{Endothelial cell markers}

Blood samples were collected from patients at baseline, and the plasma was isolated and stored at $-70{ }^{\circ} \mathrm{C}$ until analysis. Concentrations of VEGF-A, VEGF-C, soluble VEGFR-2, vascular cell adhesion molecule 1 (VCAM-1), and E-selectin were quantified using enzyme-linked immunosorbent assay kits (R \& D Systems, Minneapolis, MN). All assays were performed in duplicates.

\section{Statistical analysis}

We utilized a full analysis set (FAS), defined as all patients who met the eligibility criteria. All patients were included in the FAS. The rate of DLT, number of patients, incidence rate of all adverse drug reactions, PK, and efficacy were determined. Efficacy was assessed in terms of the RR, DCR, TTP, 
and OS. The median TTP and OS were assessed using the Kaplan-Meier method, and $95 \%$ confidence intervals (CI) were calculated. In addition, the relationship between TTP and endothelial cell markers at baseline was examined. TTP was assessed at high and low levels of endothelial cell markers. Hazard ratios (HRs) and $95 \%$ CIs were estimated by using the Cox proportional-hazards model. Changes in biomarker concentrations from baseline were evaluated using the exactpaired Wilcoxon test (two-sided). A $p$-value of $<0.05$ was considered statistically significant.

\section{Results}

Patient characteristics

Between December 2008 and June 2010, 18 patients (6 each at levels 1B, 2A, and 2B) were enrolled at National Cancer Center East, National Cancer Center, and The University of Tokyo Hospital in Japan. Baseline demographics and disease characteristics are summarized in Table 1.

Evaluation of liver function revealed that all 18 patients had Child-Pugh A cirrhosis. Of these, 7 patients (38.9\%) were at an intermediate stage and $11(61.1 \%)$ were at an advanced stage according to the Barcelona-Clinic Liver Cancer staging system [23, 24]. Seven (38.9\%) patients had previously undergone surgeries and $15(83.3 \%)$ had previously received local therapy. None of the 18 patients had ever received sorafenib.

\section{Dose delivery}

Eighteen patients (6 each at levels 1B, 2A, and 2B) received $400 \mathrm{mg}$ of TSU-68 orally per day and one of the following doses of S-1: $80 \mathrm{mg} /$ day (level 1) or $100 \mathrm{mg} /$ day (level 2). For levels $1 \mathrm{~B}-2 \mathrm{~B}$, the median relative dose intensities of TSU-68 were $70.0 \%, 76.9 \%$, and $87.1 \%$, respectively, and the corresponding values for S-1 were $70.9 \%, 75.4 \%$, and $87.0 \%$, respectively. The median duration of TSU-68 treatment was 83.3 days for level $1 \mathrm{~B}, 49.8$ days for level $2 \mathrm{~A}$, and 225.8 days for level $2 \mathrm{~B}$; the corresponding median durations of S-1 treatment were 67.8 days, 36.5 days, and 159.5 days, respectively.

Safety

Two patients at level 1B exhibited DLTs. The first patient experienced grade 3 gastrointestinal bleeding, grade 3 gastric ulcer, grade 4 hemoglobin, grade 3 nausea, and grade 3 vomiting, while the second patient had grade 2 ascites.

At level 2A, two patients of DLTs were reported, one of grade 3 fatigue and the other of grade 3 hand-foot skin reaction and grade 3 rash. However, no DLTs were observed at level $2 \mathrm{~B}$.

Table 2 shows the drug-related adverse reactions reported in at least $40 \%$ of all patients or at $\geq$ grade 3 . The most common adverse events, regardless of grade, were a decreased hemoglobin level $(83.3 \%)$, anorexia $(77.8 \%)$, hypoalbuminemia (72.2\%), localized edema (66.7\%), fatigue $(61.1 \%)$, nausea $(61.1 \%)$, a decreased platelet count $(61.1 \%)$, urine color change (55.6\%), hyperbilirubinemia (50.0\%), exfoliative rash (50.0\%), and hyperpigmentation (50.0\%). The most common grade 3 or 4 hematological toxic effect was lymphopenia $(22.2 \%)$, while the most common non-hematological toxic effects were fatigue $(22.2 \%)$, anorexia $(16.7 \%)$, and an elevated serum AST level (16.7\%).

One patient treated at level $2 \mathrm{~A}$ died of heart failure. Three patients at level $1 \mathrm{~B}$ and $2 \mathrm{~A}$ and 1 patient at level $2 \mathrm{~B}$ were hospitalized because of serious adverse events. Treatment was discontinued in 3 patients because of an adverse reaction of $\geq$
Table 1 Patient characteristics

\footnotetext{
Abbreviations: ECOG performance status, Eastern Cooperative Oncology Group performance status

HBs Ag, hepatitis B surface antigen; HCV Ab, hepatitis C antibody

BCLC stage, Barcelona-Clinic Liver Cancer staging system
}

\begin{tabular}{llllll}
\hline Variable & Category & Level 1B & Level 2A & Level 2B & Total \\
\hline Number of patients & Total & 6 & 6 & 6 & 18 \\
Sex & Male & 5 & 6 & 4 & 15 \\
Age (years) & Female & 1 & 0 & 2 & 3 \\
& Median & 72.5 & 74 & 65.5 & 71.5 \\
ECOG performance status & Range & $58-75$ & $64-85$ & $60-73$ & $58-85$ \\
& 0 & 6 & 4 & 6 & 16 \\
Viral markers & 1 & 0 & 2 & 0 & 2 \\
\multirow{3}{*}{ BCLC stage } & HBs Ag+ & 1 & 2 & 0 & 3 \\
\multirow{2}{*}{ Child-Pugh status } & HCV Ab+ & 3 & 4 & 5 & 12 \\
& Intermediate & 1 & 3 & 3 & 7 \\
\hline
\end{tabular}


Table 2 Adverse drug reactions according to grade $(\mathrm{n}=18)$ observed in the study population
Results are expressed as the worst TSU-68 or S-1-related adverse event that occurred in at least $40 \%$ of patients or were classified as grade $\geq 3$

All adverse events are documented according to the National Cancer Institute Common

Toxicity Criteria version 3.0

Abbreviations: pts, number of patients; $\mathrm{Gr} \geq 3$, grade $\geq 3$

\begin{tabular}{|c|c|c|c|c|c|c|c|c|}
\hline & \multicolumn{2}{|c|}{ Level 1B } & \multicolumn{2}{|c|}{ Level 2A } & \multicolumn{2}{|c|}{ Level 2B } & \multicolumn{2}{|c|}{ Total } \\
\hline & pts & $\mathrm{Gr} \geq 3$ & pts & $\mathrm{Gr} \geq 3$ & pts & $\mathrm{Gr} \geq 3$ & pts & $\mathrm{Gr} \geq 3$ \\
\hline Hemoglobin decrease & 4 & 2 & 6 & 0 & 5 & 0 & 15 & 2 \\
\hline Anorexia & 5 & 0 & 5 & 2 & 4 & 1 & 14 & 3 \\
\hline Hypoalbuminemia & 4 & 0 & 4 & 0 & 5 & 0 & 13 & 0 \\
\hline Localized edema & 4 & 0 & 4 & 0 & 4 & 0 & 12 & 0 \\
\hline Fatigue & 4 & 1 & 3 & 2 & 4 & 1 & 11 & 4 \\
\hline Nausea & 4 & 1 & 4 & 0 & 3 & 0 & 11 & 1 \\
\hline Platelet count decrease & 4 & 0 & 5 & 1 & 2 & 0 & 11 & 1 \\
\hline Urine color change & 4 & 0 & 3 & 0 & 3 & 0 & 10 & 0 \\
\hline Hyperbilirubinemia & 2 & 1 & 4 & 0 & 3 & 0 & 9 & 1 \\
\hline Exfoliative rash & 4 & 0 & 3 & 1 & 2 & 0 & 9 & 1 \\
\hline Skin hyperpigmentation & 2 & 0 & 3 & 0 & 4 & 0 & 9 & 0 \\
\hline Lymphopenia & 3 & 2 & 4 & 2 & 1 & 0 & 8 & 4 \\
\hline Aspartate aminotransferase level increase & 4 & 2 & 1 & 1 & 3 & 0 & 8 & 3 \\
\hline Neutrophil count decrease & 3 & 0 & 2 & 1 & 3 & 1 & 8 & 2 \\
\hline Hyponatremia & 3 & 2 & 3 & 0 & 1 & 0 & 7 & 2 \\
\hline Vomiting & 3 & 1 & 2 & 0 & 2 & 0 & 7 & 1 \\
\hline Alanine aminotransferase level increase & 2 & 1 & 2 & 1 & 1 & 0 & 5 & 2 \\
\hline Hypokalemia & 2 & 1 & 1 & 1 & 0 & 0 & 3 & 2 \\
\hline Hand-foot skin reaction & 0 & 0 & 2 & 1 & 1 & 0 & 3 & 1 \\
\hline Pleural effusion & 1 & 0 & 1 & 1 & 0 & 0 & 2 & 1 \\
\hline Gastric hemorrhage & 1 & 1 & 0 & 0 & 0 & 0 & 1 & 1 \\
\hline Gastric ulcer & 1 & 1 & 0 & 0 & 0 & 0 & 1 & 1 \\
\hline Liver abscess & 0 & 0 & 0 & 0 & 1 & 1 & 1 & 1 \\
\hline Thrombosis & 0 & 0 & 1 & 1 & 0 & 0 & 1 & 1 \\
\hline Blood glucose decrease & 1 & 1 & 0 & 0 & 0 & 0 & 1 & 1 \\
\hline Myocardial ischemia & 0 & 0 & 0 & 0 & 1 & 1 & 1 & 1 \\
\hline Pericardial effusion & 0 & 0 & 1 & 1 & 0 & 0 & 1 & 1 \\
\hline Cardiac failure & 0 & 0 & 1 & 1 & 0 & 0 & 1 & 1 \\
\hline
\end{tabular}

grade 3 , including grade 4 pericardial effusion/grade 4 pleural effusion, grade 3 fatigue, and grade 3 hand-foot reactions/ grade 3 rash.

\section{PK}

The PK parameters of TSU-68 after co-administration with S-1 were the same for level 1B and 2A, as shown in Fig. 2. In addition, these PK parameters were similar to those for TSU68 administered alone.

The PK parameters of S-1 components and 5-FU at level $2 \mathrm{~A}$ were slightly higher than the corresponding values at level $1 \mathrm{~B}$, as expected after the dose increase. The $\mathrm{C}_{\max }$ and AUC of 5-FU, CDHP, and Oxo were not markedly different between day 1 and day 8 , while those of FT were higher on day 8 than on day 1 because of accumulated exposure with repeated administration of S-1.

\section{Efficacy}

The anti-tumor effect of TSU-68 and S-1 was evaluated in the 18 patients included in the FAS (Table 3). At level 1B, 1 of the 6 patients showed partial response (PR), corresponding to a response rate of $16.7 \%$. At level $2 \mathrm{~B}, 4$ patients had $\mathrm{PR}$, corresponding to a response rate of $66.7 \%$.

The median TTP was 3.9 months at level 1B, 2.4 months at level 2A, 8.0 months at level 2B, and 5.3 months for all patients (Fig. 3). OS was evaluated for 18 patients in the FAS and was 10.7 months at level $1 \mathrm{~B}, 14.9$ months at level 2A, 16.3 months at level 2B, and 12.8 months for all patients (Fig. 3).

Endothelial cell markers

Biomarker analysis showed that TTP was significantly longer in patients with low VCAM-1 levels than that in other patients 

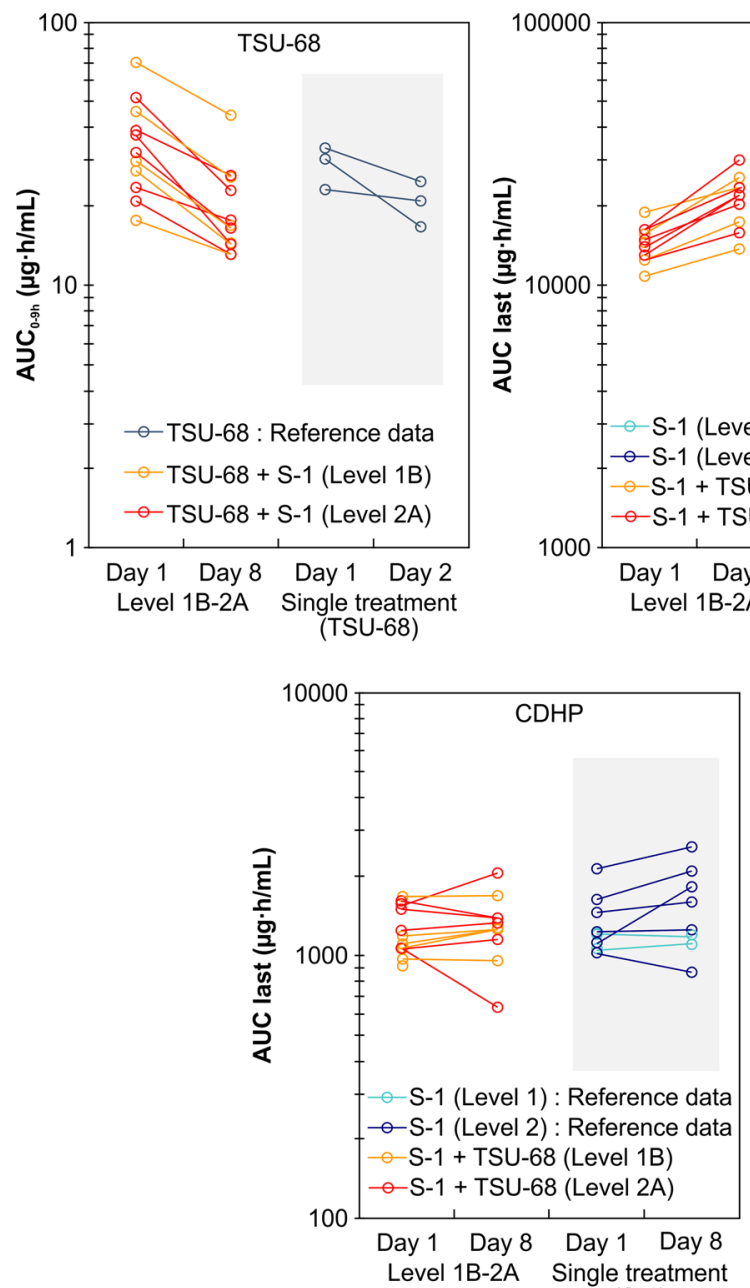

(S-1)

Fig. 2 Pharmacokinetics analysis of data from 12 patients at levels 1B and $2 \mathrm{~A}$. The results indicated that the areas under the curve (AUC) of TSU-68 and 5-FU were unlikely to be affected by the combined administration of TSU-68 plus S-1. The AUC of FT appeared to decrease on the

(hazard ratio, 0.26; $95 \%$ CI, 0.08-0.85; $p=0.018$ ) (Table 4). No significant difference was observed with other endothelial cell markers examined.

\section{Discussion}

In the present study, we determined the recommended dose of the TSU-68 plus S-1 combination in patients with advanced HCC based on the frequency of associated DLTs. We also investigated the safety, tolerability, PK, and efficacy of the TSU-68 plus S-1 combination in our study population. For combination therapy, it is important to achieve efficacy without worsening of PK parameters or adverse drug reactions. Investigation of molecular markers in TSU-68 plus S-1 combination therapy merits additional research.

We determined the safety of several treatment levels by examining the frequency of associated DLTs. Such events

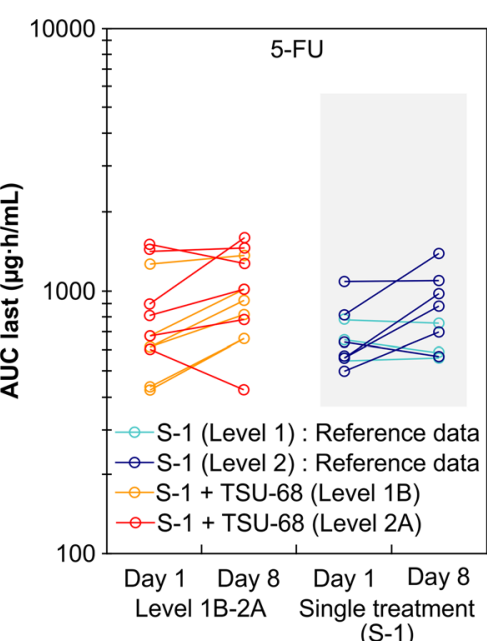

(S-1)

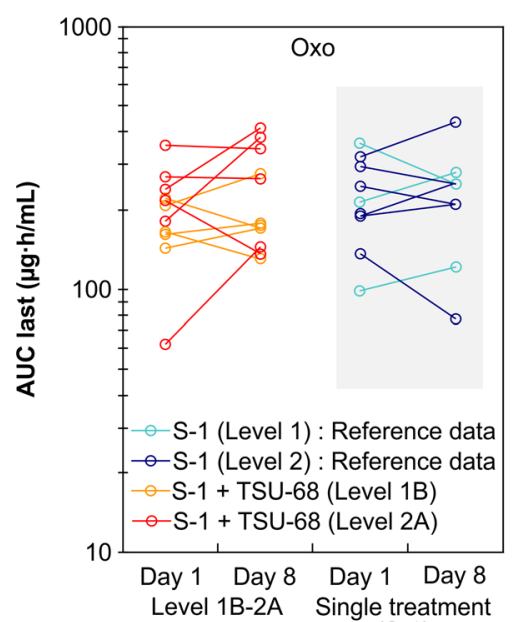

(S-1)

administration of TSU-68 plus S-1 compared to that on S-1 alone. However, other components were not affected by the administration of TSU-68 plus S-1 in combination compared to that of S-1 alone

were observed in 2 patients at level 1B (1 with gastrointestinal bleeding, gastric ulcer, hemoglobin, nausea, and vomiting, and the other 1 with ascites) and in 2 patients at level 2A (1 with fatigue and the other 1 with hand-foot skin reaction and rash). However, at level 2B, none of the patients demonstrated DLTs. With regard to adverse drug reactions of grade 3 or higher severity, 17 events were noted at levels $1 \mathrm{~B}$ and $2 \mathrm{~A}$ and 5 events were noted at level 2B. Overall, treatment at level 2B resulted in the least severe DLTs, less serious adverse events, and fewer adverse reactions of grade 3 or higher severity.

When we compared the adverse drug reaction incidence in this trial with those reported in independent trials for TSU-68 [9] and S-1 [12], in which each agent was administered alone to patients with HCC, we found that TSU-68 plus S-1 combination therapy did not increase adverse drug reactions. Compared to the trial in which TSU-68 was administered alone, the incidences of anorexia, localized edema, fatigue, nausea, skin pigmentation, hemoglobin level decrease, hypoalbuminemia, 
Table 3 Efficacy results in all patients

\begin{tabular}{|c|c|c|c|}
\hline & $\begin{array}{l}\text { Level 1B } \\
(n=6)\end{array}$ & $\begin{array}{l}\text { Level 2A } \\
(n=6)\end{array}$ & $\begin{array}{l}\text { Level 2B } \\
(\mathrm{n}=6)\end{array}$ \\
\hline Complete response, n (\%) & $0(0.0)$ & $0(0.0)$ & $0(0.0)$ \\
\hline Partial response, n (\%) & $1(16.7)$ & $0(0.0)$ & $4(66.7)$ \\
\hline Stable disease, $\mathrm{n}(\%)$ & $2(33.3)$ & $3(50.0)$ & $1(16.7)$ \\
\hline Progressive disease, $\mathrm{n}(\%)$ & $3(50.0)$ & $3(50.0)$ & $1(16.7)$ \\
\hline Not evaluated, n (\%) & $0(0.0)$ & $0(0.0)$ & $0(0.0)$ \\
\hline Response rate, n (\%) & $1(16.7)$ & $0(0.0)$ & $4(66.7)$ \\
\hline $95 \%$ CI (\%) & $0.4-64.1$ & $0.0-45.9$ & $22.3-95.7$ \\
\hline Disease control rate, $\mathrm{n}(\%)$ & $3(50.0)$ & $3(50.0)$ & $5(83.3)$ \\
\hline $95 \%$ CI (\%) & $11.8-88.2$ & $11.8-88.2$ & $35.9-99.6$ \\
\hline Median TTP (month) & 3.9 & 2.4 & 8 \\
\hline 95 \% CI (month) & $2.5-6.3$ & $1.9--$ & $5.3-12.2$ \\
\hline MST (month) & 10.7 & 14.9 & 16.3 \\
\hline 95 \% CI (month) & $5.7--$ & $11.3-20.8$ & $11.6--$ \\
\hline
\end{tabular}

Abbreviations: n, number; TTP, time to progression; MST, median survival time

thrombopenia, and hyperbilirubinemia were higher in the present study by more than $20 \%$. However, compared to the trial in which S-1 was administered alone, the incidences of localized edema and nausea in the present study were higher by more than $20 \%$ while those of some other adverse drug reactions were lower by more than $20 \%$. Nevertheless, there was no difference in the adverse drug reaction rate between the TSU-68 and S- 1 combination therapy and TSU-68 or S-1 administered alone. The common adverse drug reactions of the TSU-68 plus S-1 combination were mild in severity (grades 1-2). Our results demonstrated that the TSU-68 plus S-1 combination was well tolerated in patients with $\mathrm{HCC}$.

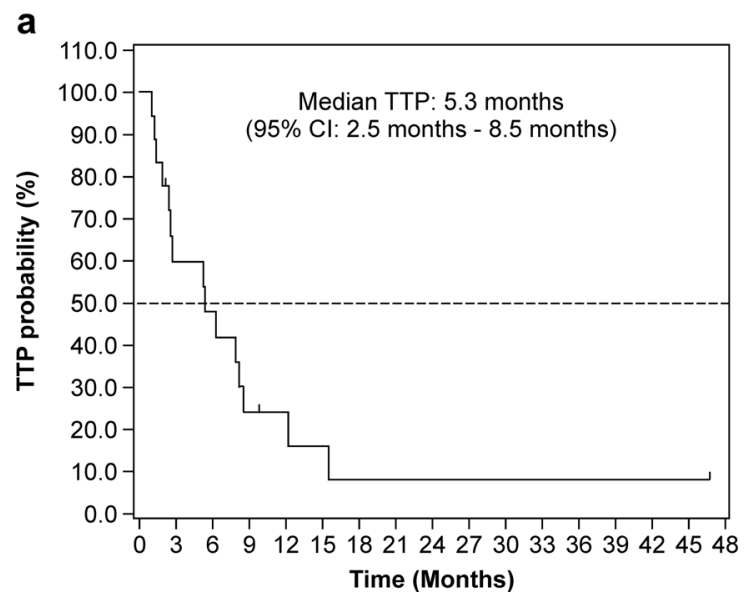

We compared the PK data on TSU-68 plus S-1 combination therapy with the PK data on S-1 or TSU-68 alone $[9,12]$. Our results suggested that TSU-68 PK parameters were unlikely to be affected by co-administration with S-1. The $\mathrm{C}_{\max }$ and AUC of TSU-68 on day 8 were lower than those on day 1 for cytochrome P450 1A2, as has been reported previously [9]. For S-1, exposure to FT after repeated co-administration with TSU-68 tended to be lower than that reported previously for S-1 administered alone [12]. However, there were no apparent differences in the PK parameters of 5-FU, CDHP, and Oxo between the two studies. Our data indicated that the PK parameters of TSU-68 and S-1 were independent and hence, unaffected by combined administration, with the exception of exposure to FT.

Next, we compared the effectiveness of TSU-68 and S-1 administered alone and in combination. TTP and OS for the 18 patients receiving TSU-68 plus S-1 combination therapy were 5.3 months and 12.8 months, and 8.0 months and 16.3 months at level $2 \mathrm{~B}$, respectively. On the other hand, the progression-free survival (PFS) and OS for patients receiving S-1 alone were 3.7 months and 16.6 months, respectively, and the TTP and OS for patients with TSU-68 alone were 2.1 months and 13.1 months. Therefore, the efficacy of the combination therapy at level $2 \mathrm{~B}$ treatment was better than that of either TSU-68 or S-1 treatment alone.

We also compared the efficacy of the TSU-68 plus S-1 combination administered at level $2 \mathrm{~B}$ in this study to that of sorafenib plus S-1 [14] and sorafenib plus Dox [15]. The PFS was 3.9 months and 6.0 months for sorafenib plus S-1 and sorafenib plus Dox, respectively, while the corresponding OS was 10.4 months and 13.7 months, respectively. Therefore, the efficacy of level 2B treatment was better than that of the other two reported combinations.

Taken together, our results indicate that the TSU-68 plus S-1 combination therapy is safe and efficacious; nevertheless, further investigation of the treatment at level $2 \mathrm{~B}$, in particular, is warranted.

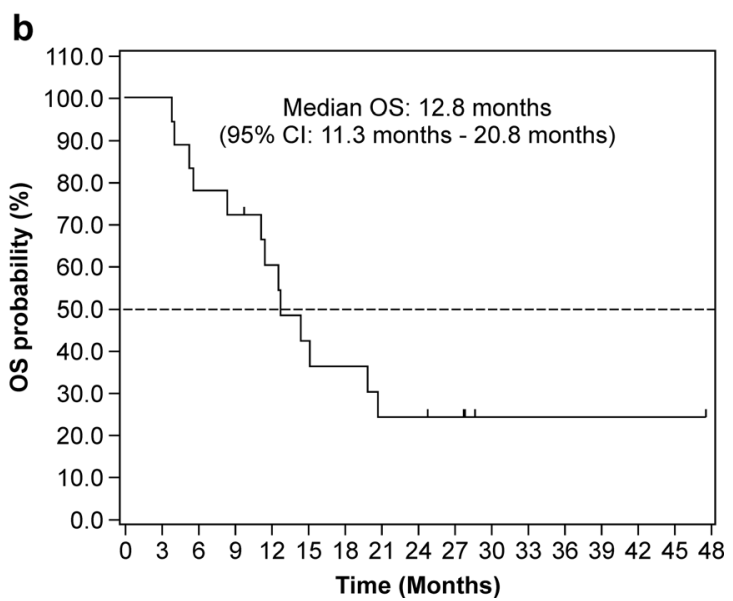

Fig. 3 Kaplan-Meier analysis demonstrating the time to progression (a) and overall survival (b) of advanced hepatocellular carcinoma patients treated with the TSU-68 plus S-1 combination 
Table 4 Biomarker analysis

\begin{tabular}{|c|c|c|c|c|c|c|}
\hline & Median & $\begin{array}{l}<\text { Median conc. } \\
\text { Median TTP (days) }\end{array}$ & $\begin{array}{l}\geq \text { Median conc. } \\
\text { Median TTP (days) }\end{array}$ & Hazard ratio & $95 \% \mathrm{CI}$ & $p$ value ${ }^{*}$ \\
\hline VCAM-1 & 1,310 & 372 & 77 & 3.89 & $1.17-12.9$ & 0.018 \\
\hline ELAM-1 & 52 & 193 & 157 & 1.14 & $0.41-3.18$ & 0.799 \\
\hline PDGF-BB & 1793.1 & 79 & 239 & 0.81 & $0.28-2.37$ & 0.704 \\
\hline Total PAI-1 & 15 & 193 & 157 & 1.99 & $0.68-5.84$ & 0.205 \\
\hline FGF acidic & 31.3 & - & 160 & - & - & - \\
\hline bFGF & 10 & - & 160 & - & - & - \\
\hline PDGF-AA & 418.55 & 157 & 239 & 1.09 & $0.39-3.03$ & 0.865 \\
\hline VEGF & 51.5 & 157 & 239 & 0.53 & $0.18-1.6$ & 0.255 \\
\hline sVEGFR-2 & 7170.35 & 160 & 193 & 1.01 & $0.36-2.8$ & 0.992 \\
\hline VEGF-C & 1092.1 & 79 & 239 & 0.76 & $0.27-2.1$ & 0.594 \\
\hline VEGFR-3 & 8063.45 & 193 & 160 & 2.1 & $0.67-6.56$ & 0.192 \\
\hline
\end{tabular}

*Log-rank test

Abbreviations: conc, concentration; TTP, time to progression

VCAM-1, vascular cell adhesion molecule 1

ELAM-1, endothelial-leukocyte adhesion molecule 1

PDGF-BB, platelet-derived growth factor BB

PAI-1, plasminogen activator inhibitor 1

FGF, fibroblast growth factor

bFGF, basic fibroblast growth factor

PDGF-AA, platelet-derived growth factor AA

VEGF, vascular endothelial growth factor

sVEGFR-2, soluble vascular endothelial growth factor receptor 2

VEGF-C, vascular endothelial growth factor $\mathrm{C}$

VEGFR-3, vascular endothelial growth factor receptor 3

Furthermore, we evaluated expression of endothelial cell markers in patients receiving the TSU-68 plus S- 1 combination therapy. VCAM-1 is aberrantly expressed in breast cancer cells and mediates pro-metastatic tumor-stroma interactions $[25,26]$. In HCC, serum VCAM-1 level appears to reflect the severity of the underlying chronic liver disease rather than the tumor status $[27,28]$, and low preoperative serum VCAM-1 levels are predictive of better disease-free survival after surgery [28]. Our results suggest that the VCAM-1 level may be used as a predictive factor for TSU-68 plus S-1 combination therapy. However, these data are preliminary and further research will be needed to confirm the relationship between VCAM-1 and prognosis in TSU-68 plus S-1 combination therapy.

In conclusion, our findings reveal that the TSU-68 plus S-1 recommended dose for advanced HCC is $400 \mathrm{mg}$ /day TSU-68 and $100 \mathrm{mg} /$ day S-1 for 4 weeks followed by 2 -week rest. TSU-68 plus S-1 combination was well tolerated and had favorable efficacy in patients with advanced HCC. Biomarker analysis showed that VCAM-1 may be a possible predictive marker for response. Further study is necessary to confirm whether TSU-68 plus S- 1 combination therapy is a therapeutic option for advanced HCC and if VCAM-1 is a possible predictive marker for response.

Acknowledgments We thank the Data Monitoring Committee members Yutaka Ariyoshi, Yuh Sakata, and Tomohide Tamura for their contributions. This trial was supported by Taiho Pharmaceutical Co., Ltd.

Conflict of Interest All authors have no conflicts of interest to declare.

Open Access This article is distributed under the terms of the Creative Commons Attribution License which permits any use, distribution, and reproduction in any medium, provided the original author(s) and the source are credited.

\section{References}

1. Parkin DM, Bray F, Ferlay J, Pisani P (2005) Global cancer statistics 2002. CA Cancer J Clin 55:74-108

2. Llovet JM, Ricci S, Mazzaferro V et al (2008) Sorafenib in advanced hepatocellular carcinoma. N Engl J Med 359:378-390

3. Cheng AL, Kang YK, Chen Z et al (2009) Efficacy and safety of sorafenib in patients in the Asia-Pacific region with advanced 
hepatocellular carcinoma: a phase III randomised, double-blind, placebo-controlled trial. Lancet Oncol 10:25-34

4. Laird AD, Vajkoczy P, Shawver LK et al (2000) SU6668 is a potent antiangiogenic and antitumor agent that induces regression of established tumors. Cancer Res 60:4152-4160

5. Solorzano CC, Jung YD, Bucana CD et al (2001) In vivo intracellular signaling as a marker of antiangiogenic activity. Cancer Res 61: 7048-7051

6. Kuenen BC, Giaccone G, Ruijter R et al (2005) Dose-finding study of the multitargeted tyrosine kinase inhibitor SU6668 in patients with advanced malignancies. Clin Cancer Res 11: 6240-6246

7. Yorozuya K, Kubota T, Watanabe M et al (2005) TSU-68 (SU6668) inhibits local tumor growth and liver metastasis of human colon cancer xenografts via anti-angiogenesis. Oncol Rep 14:677-682

8. Naumova E, Ubezio P, Garofalo A et al (2006) The vascular targeting property of paclitaxel is enhanced by SU6668, a receptor tyrosine kinase inhibitor, causing apoptosis of endothelial cells and inhibition of angiogenesis. Clin Cancer Res 12:1839-1849

9. Kanai F, Yoshida H, Tateishi R et al (2011) A phase I/II trial of the oral antiangiogenic agent TSU-68 in patients with advanced hepatocellular carcinoma. Cancer Chemother Pharmacol 67:315-324

10. Inaba Y, Kanai F, Aramaki T et al (2013) A randomised phase II study of TSU-68 in patients with hepatocellular carcinoma treated by transarterial chemoembolisation. Eur J Cancer 49:2832-2840

11. Shirasaka T, Shimamoto Y, Fukushima M (1993) Inhibition by oxonic acid of gastrointestinal toxicity of 5-fluorouracil without loss of its antitumor activity in rats. Cancer Res 53:4004-4009

12. Furuse J, Okusaka T, Kaneko S et al (2010) Phase I/II study of the pharmacokinetics, safety and efficacy of S-1 in patients with advanced hepatocellular carcinoma. Cancer Sci 101:2606-2611

13. Finn RS, Poon RT, Yau T et al (2013) Phase I study investigating everolimus combined with sorafenib in patients with advanced hepatocellular carcinoma. J Hepatol 59:1271-1277

14. Lee SJ, Lee J, Park SH et al (2012) Phase 1 trial of S-1 in combination with sorafenib for patients with advanced hepatocellular carcinoma. Invest New Drugs 30:1540-1547

15. Abou-Alfa GK, Johnson P, Knox JJ et al (2010) Doxorubicin plus sorafenib vs doxorubicin alone in patients with advanced hepatocellular carcinoma: a randomized trial. JAMA 304: 2154-2160
16. Hsu CH, Shen YC, Lin ZZ et al (2010) Phase II study of combining sorafenib with metronomic tegafur/uracil for advanced hepatocellular carcinoma. J Hepatol 53:126-131

17. Petrini I, Lencioni M, Ricasoli M et al (2012) Phase II trial of sorafenib in combination with 5-fluorouracil infusion in advanced hepatocellular carcinoma. Cancer Chemother Pharmacol 69:773-780

18. Hsu CH, Yang TS, Hsu C et al (2010) Efficacy and tolerability of bevacizumab plus capecitabine as first-line therapy in patients with advanced hepatocellular carcinoma. Br J Cancer 16:981-986

19. Kudo M, Imanaka K, Chida N et al (2011) Phase III study of sorafenib after transarterial chemoembolisation in Japanese and Korean patients with un-resectable hepatocellular carcinoma. Eur J Cancer 47:2117-2127

20. Lencioni R, Llovet JM, Han G et al (2012) Sorafenib or placebo in combination with transarterial chemoembolization (TACE) with doxorubicin-eluting beads (DEBDOX) for intermediate-stage hepatocellular carcinoma (HCC): phase II, randomized, double-blind SPACE trial. J Clin Oncol 30:154

21. Wörns MA (2013) Systemic therapy and synergies by combination. Dig Dis 31:104-111

22. Therasse P, Eisenhauer EA, Verweij J (2006) RECIST revisited: a review of validation studies on tumour assessment. Eur J Cancer 42: 1031-1039

23. Llovet JM, Bruix J (2008) Novel advancements in the management of hepatocellular carcinoma. J Hepatology 48:20-37

24. Bruix J, Sherman M (2005) Management of hepatocellular carcinoma. Hepatology 42:1208-1236

25. Chen Q, Zhang XH, Massagué J (2011) Macrophage binding to receptor VCAM-1 transmits survival signals in breast cancer cells that invade the lungs. Cancer Cell 20:538-549

26. Lu X, Mu E, Wei Y et al (2011) VCAM-1 promotes osteolytic expansion of indolent bone micrometastasis of breast cancer by engaging $\alpha 4 \beta 1$-positive osteoclast progenitors. Cancer Cell 20: $701-714$

27. Diaz-Sanchez A, Matilla A, Nuñez O et al (2013) Serum level of soluble vascular cell adhesion molecule in patients with hepatocellular carcinoma and its association with severity of liver disease. Ann Hepatol 12:236-247

28. Ho JW, Poon RT, Tong CS, Fan ST (2004) Clinical significance of serum vascular cell adhesion molecule-1 levels in patients with hepatocellular carcinoma. World J Gastroenterol 10:2014-2018 